WSRC-TR--91-474-1

DE92 014237

\title{
F/H AREA ETF EFFLUENT (H-016 OUTFALL) CERIODAPHNIA SURVIVAL/REPRODUCTION TEST, TEST DATE: DECEMBER 12, 1990 (U)
}

by

W. L. Specht, et al.

Westinghouse Savannah River Company

Savannah River Laboratory

Aiken, South Carolina 29808

A Technical Report for Publication

\section{DISCLAIMER}

\begin{abstract}
This report was prepared as an account of work sponsored by an agency of the United States Government. Neither the United States Government nor any agency thereof, nor any of their employees, makes any warranty, express or implied, or assumes any legal liability or responsibility for the accuracy, completeness, or usefulness of any information, apparatus, product, or process disclosed, or represents that its use would not infringe privately owned rights. Reference herein to any specific commercial product, process, or service by trade name, trademark, manufacturer, or otherwise does not necessarily constitute or imply its endorsement, recommendation, or favoring by the United States Government or any agency thereof. The views and opinions of authors expressed herein do not necessarily state or reflect those of the United States Government or any agency thereof.
\end{abstract}

This paper was prepared in connection with work done under Contract No. DE-AC09-89SR18035 with the U.S. Department of Energy. By acceptance of this paper, the publisher and/or recipient acknowledges the U.S. Government's right to retain a nonexclusive, royalty-free license in and to any copyright covering this paper, along with the right to reproduce and to authorize others to reproduce all or part of the copyrighted paper. 
Derivative Classifier DBonowe-Shedone

D. B. Moore-Shedrow, Section Manager

Authorized Derivative Classifer

MAY 261992

F/H AREA ETF EFFLUENT (H-016 OUTFALL)

CERIODAPHNIA SURVIVAL/REPRODUCTION TEST, TEST DATE: DECEMBER 12, 1990

WSRC Technical Representative: W. L. Specht

$\begin{aligned} & \text { Approved by }: \frac{\text { Demome-Smedow }}{\text { D. B. Moore-Shedrow, Section Manager }} \\ & \text { Environmental Sciences Section } \\ & \text { Savannah River Laboratory }\end{aligned}$

Publication Date: August 1991

WESTINGHOUSE SAVANNAH RIVER COMPANY

SAVANNAH RIVER SITE

AIKEN, SC 29808

Prepared for the U.S. Department of Energy under Contract No.

DE-ACO9-89SR18035 


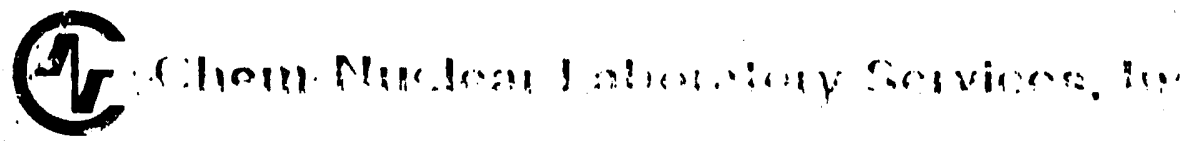

$1,1+11,+1: 1,11$

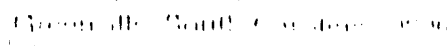

(,..............

Ceriodaphnia SURVIVAL/REPRODUCTION TEST

Sample Identification: $\quad$ H - F Area

Client: Savannah River Site

Location: Aiken, SC

Test Date: December 12, 1990 


\section{INTERPRETATION OF RESULTS}

This toxicity test was conducted to determine if the effluent affects the survival or reproduction of the test organisms during a seven day period. The test involved exposing the test organisms to a series of dilutions of the effluent. At each dilution the survival and reproduction of ten test organisms was recorded. Each effluent dilution was compared to a control set of test organisms. Survival data were analyzed by Fisher's Exact Test and the Trimmed Spearman-Karber method to determine the effluent concentration necessary to cause statistically significant $(p=0.05)$ mortality. Reproduction data was analyzed for normality, homogeneity of variance and equality of replicates among dilutions to determine appropriate statistical test for analysis of statistical differences in reproduction among dilutions. Results are summarized as follows:

Acute Toxicity:

Probit Analysis

LC5O= $44.38 \%$ Effluent
Fisher's Exact Test
NOEC $=\quad \begin{array}{r}30.08 \text { Effluent } \\ \text { LOEC }= \\ 100.08 \text { Effluent }\end{array}$

95\% Confidence Limits Lower Upper

$27.47 \quad 71.71$

Chronic Toxicity

Bonferroni's $t$-Test

(NOEC) No Observed Effect Conc.:

30.08

(LOEC) Lowest Observed Effect Conc.:

$100.0 \%$

Chronic value:

54.968

$(p=0.05)$ 
Three effluent samples were collected by Chem-Nuclear Laboratory Services by on-site personnel and were transported by either same-day or overnight delivery. Samples were collected in $41 \mathrm{~L}$ plastic cubitainers.

Client: Savannah River Site

Sample Identification: H - F Area

start Date: December 12, 1990

$$
\begin{array}{lll}
\# 1 & \text { Sample } & \text { Sample } \\
\# 2 & \$ 3
\end{array}
$$

\begin{tabular}{|c|c|c|c|}
\hline $\begin{array}{l}\text { Lab \# } \\
\text { Type }\end{array}$ & $\begin{array}{r}\text { BI0730-1 } \\
\text { grab }\end{array}$ & $\begin{array}{r}\text { BIO730-2 } \\
\text { grab }\end{array}$ & $\begin{array}{r}\text { BIO730-3 } \\
\text { grab }\end{array}$ \\
\hline Date sampled & $12 / 11 / 90$ & $12 / 14 / 90$ & $12 / 17 / 90$ \\
\hline Time sampled & $9: 50 \mathrm{AM}$ & $9: 40$ AM & $12: 45 \mathrm{PM}$ \\
\hline Days of use & $0,1,2$ & 3,4 & 5,6 \\
\hline Maximum hold time before use: & $36 \mathrm{hrs}$ & 72 hrs & 72 hrs \\
\hline
\end{tabular}

Effluent Field

Parameters

Sample

Sample

Sample

D.O. (ppm)

$\mathrm{pH}$

Temp (C)

Res Cl (ppm)

$6 \longdiv { 8 7 }$

$15^{\circ} \mathrm{C}$

$<0.05 \star$
$\# 2$

11.0

7.00

$16^{\circ} \mathrm{C}$

$0.05 *$
$\# 3$

11.5

6.95

$17.5^{\prime} \mathrm{C}$

$0.05 *$

* By DPL Method for Residual Chlorine Detection 


\section{Client: Savannah River Site}

Start Date: December 12, 1990

Test Type: 7 Day Chronic Toxicity - Reproduction Test

Test Location: Chem-Nuclear Laboratory Services

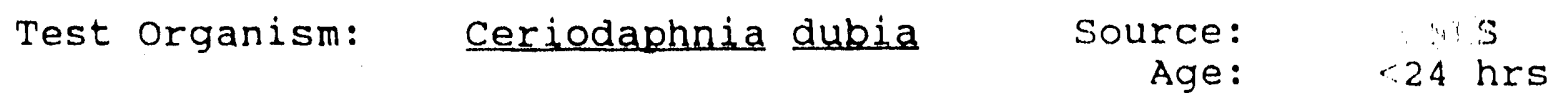

Test procedure: Short-Term Methods for Estimating the Chronic Toxicity of Effluents and Receiving waters to Freshwater Organisms. 2nd Edition. EPA 600/4-89/001 Method 1002

Test vessels: $\quad 30 \mathrm{ml}$ plastic beakers

Amount Test Soln: $15 \mathrm{ml} /$ replicate

\# Replicates/Concentration: 10

Test organisms/Replicate:

10 (test vessels randomized)

\# of Test Concentrations Used (excluding control):

Test Solution Renewal: Daily

Dilutions Used: $0,1,3,10,30$, and $100 \%$

Food Source: $\quad 0.10 \mathrm{ml}$ Selenastrum $\left(3.1-3.5 \times 10^{7} \mathrm{cells} / \mathrm{ml}\right)$ and $0.05 \mathrm{ml}$ YCT daily/ test vessel

Test Performed By:

Test Results and Report Prepared By:
Joel Stephens

Analytical Biologist

Gaol a. Stopen

Joel Stephens

Analytical Biologist

Gee a. stepien in in 


\section{RESULTS}

Client: Savannah River site

Sample Identification: H - F Area

Start Date: December 12, 1990

CHRONIC TOXICITY - Daily Reproduction Totals

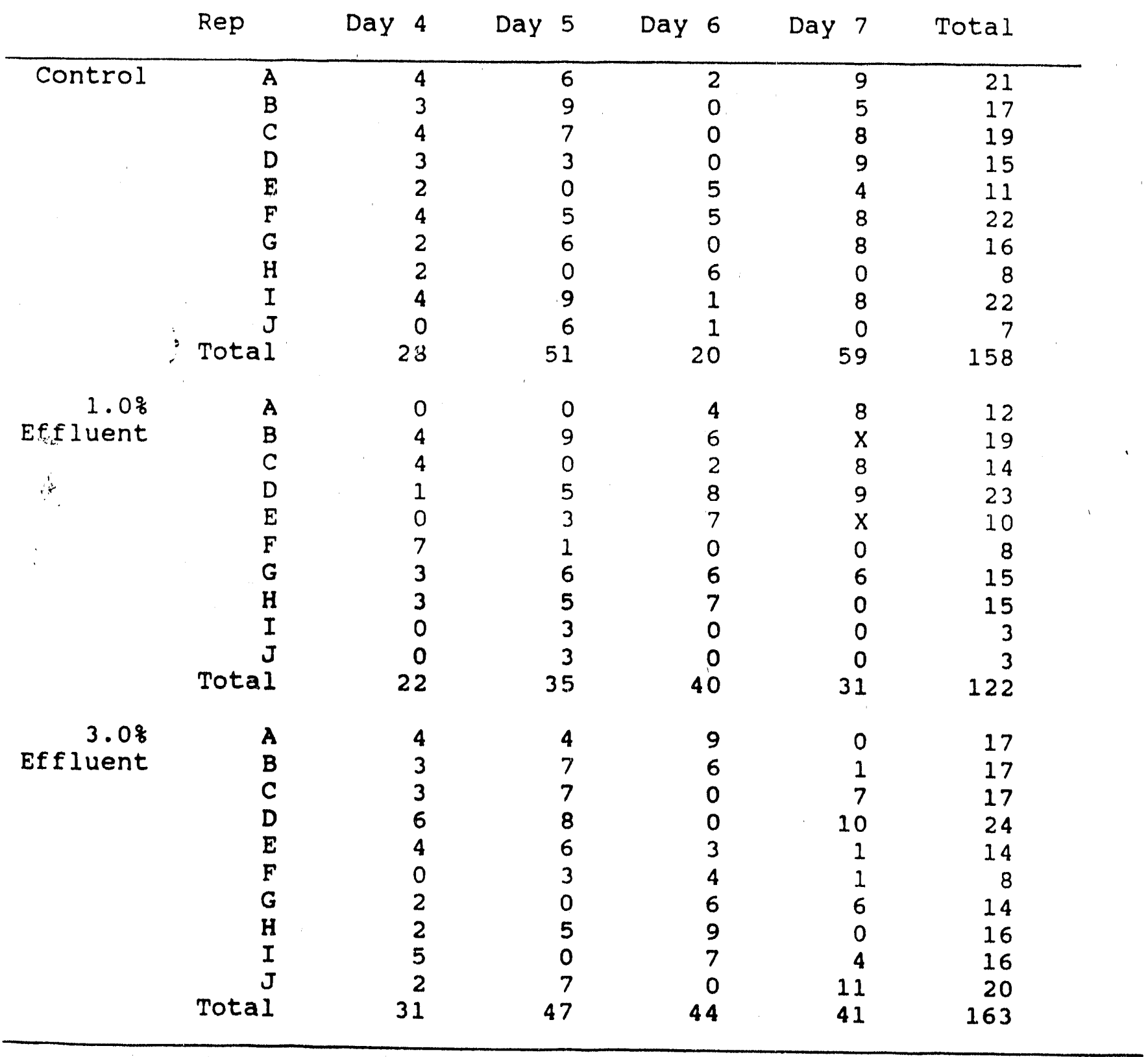


Client: Savannah River site Sample Identification: H - F Area Start Date: December 12, 1990

CHRONIC TOXICITY - Daily Reproduction Totals

\begin{tabular}{|c|c|c|c|c|c|c|}
\hline & Rep & Day 4 & Day j & Day 6 & Day 7 & Total \\
\hline \multirow{11}{*}{$\begin{array}{r}10.0 \% \\
\text { Effluent }\end{array}$} & $\bar{A}$ & 0 & 0 & 0 & $M$ & 0 \\
\hline & B & 0 & 4 & 0 & 0 & 4 \\
\hline & C & 0 & 0 & $M$ & $\mathrm{X}$ & 0 \\
\hline & $D$ & 0 & 0 & 7 & $x$ & 7 \\
\hline & $E$ & 0 & 6 & 2 & 2 & 10 \\
\hline & $\mathrm{F}$ & 2 & 8 & 0 & 3 & 13 \\
\hline & G & 0 & 4 & 3 & 0 & 7 \\
\hline & $\mathrm{H}$ & 4 & 0 & 7 & 0 & 11 \\
\hline & $I$ & 4 & 7 & 0 & 13 & 24 \\
\hline & $\mathrm{J}$ & 1 & 3 & 8 & 5 & 17 \\
\hline & Total & 11 & 32 & 27 & 23 & 93 \\
\hline \multirow{11}{*}{$\begin{array}{r}30.0 \% \\
\text { Effluent }\end{array}$} & $A$ & 0 & 4 & 0 & 0 & 4 \\
\hline & B & 1 & 7 & 13 & 0 & 21 \\
\hline & $C$ & 2 & 10 & 0 & 5 & 17 \\
\hline & D & 0 & 3 & 5 & $X$ & 8 \\
\hline & $E$ & 1 & 4 & 7 & 0 & 12 \\
\hline & $F$ & 6 & 0 & 8 & 12 & 26 \\
\hline & G & 0 & 0 & 7 & 0 & 7 \\
\hline & $\mathrm{H}$ & 0 & 4 & 6 & 5 & 15 \\
\hline & $I$ & 2 & 7 & 8 & 0 & 17 \\
\hline & $J$ & 1 & 3 & 0 & 8 & 12 \\
\hline & Total & $1 \overline{3}$ & 42 & 54 & 30 & 139 \\
\hline \multirow{11}{*}{$\begin{array}{r}100.0 \% \\
\text { Effluent }\end{array}$} & $A$ & $\mathrm{x}$ & $x$ & $X$ & $x$ & 0 \\
\hline & $\mathrm{B}$ & $x$ & $\mathrm{x}$ & $x$ & $x$ & 0 \\
\hline & $C$ & $x$ & $x$ & $x$ & $\ddot{x}$ & 0 \\
\hline & D & $\mathrm{X}$ & $x$ & $\mathrm{X}$ & $x$ & 0 \\
\hline & $\mathbf{E}$ & $x$ & $\mathrm{X}$ & $\mathrm{x}$ & $\mathrm{X}$ & 0 \\
\hline & $F$ & $x$ & $x$ & $x$ & $\mathrm{X}$ & 0 \\
\hline & G & $\mathrm{X}$ & $x$ & $\mathrm{x}$ & $x$ & 0 \\
\hline & $\mathrm{H}$ & $x$ & $\mathrm{X}$ & $\mathrm{X}$ & $x$ & 0 \\
\hline & $I$ & $x$ & $\mathrm{X}$ & $\mathrm{x}$ & $\mathrm{x}$ & 0 \\
\hline & $\mathrm{J}$ & $x$ & $x$ & $\mathrm{X}$ & $x$ & 0 \\
\hline & Total & 0 & 0 & 0 & 0 & 0 \\
\hline
\end{tabular}

\footnotetext{
$x$ represents the mortality of the test organism.
} 


\title{
RESULISS
}

Client: Savannah River site

Sample Identification: H - F Area

Start Date: December 12, 1990

SURVIVAL DATA (At Test Termination)

$\begin{array}{llllll}0.0 \% & 1 \% & 3 \% & 10 \% & 30 \% & 100 \%\end{array}$

\begin{tabular}{|c|c|c|c|c|c|c|}
\hline Survival & $\begin{array}{l}10 \\
100 \%\end{array}$ & $\begin{array}{c}8 \\
808\end{array}$ & $\begin{array}{l}10 \\
1008\end{array}$ & $\begin{array}{c}8 \\
80 \%\end{array}$ & $\begin{array}{c}9 \\
908\end{array}$ & $\begin{array}{l}0 \\
0 \%\end{array}$ \\
\hline $\begin{array}{l}\text { Mortality } \\
(7 \text { day })\end{array}$ & $\begin{array}{l}0 \\
0 \%\end{array}$ & $\begin{array}{c}2 \\
208\end{array}$ & $\begin{array}{l}0 \\
08\end{array}$ & $\begin{array}{c}2 \\
20 \%\end{array}$ & $\begin{array}{c}1 \\
10 \%\end{array}$ & $\begin{array}{l}10 \\
1008\end{array}$ \\
\hline \# Lost & 0 & 0 & 0 & 0 & 0 & 0 \\
\hline \# Males & 0 & 0 & 0 & 2 & 0 & 0 \\
\hline Bad Brood & 0 & 0 & 0 & 0 & 0 & 0 \\
\hline
\end{tabular}

SURVIVAL - DATA ANALYSIS

Spearman-Karber

\section{5: Confidence Limits Lower Upper}

$27.47 \quad 71.71$

\begin{abstract}
LC50 $=44.38$ \& Effluent
\end{abstract}
Fisher's Exact Test:

NOEC $=\quad 30.0 \%$ Effluent

LOEC $=100.0 \%$ Effluent 


\section{RESULTS}

Client: Savannah River site Sample Identification: H - F Area Start Date: December 12, 1990

CHRONIC TOXICITY - Statistical Analysis

Bonferroni's t-Test

Conc.

$0.0 \%$

$1.0 \%$

$3.0 \%$

$10.0 \%$

$30.0 \%$

$100.0 \%$

Average Young
per Female

15.8

12.2

16.3

11.6

13.9

0.0

Std. Dev.

5.55

6.44

4.14

6.41

6.71

0

Calculated

"t" value

$-1.364 \quad-0.189$

1.491

$100 \%$

Critical

"t" Value

2.324

Reproduction

significantly less

no

no

no

no

yes

than control?

( 958 confidence)

\section{8 conf.}

(NOEC) No Observed Effect Conc.:

$30.0 \%$

(LOEC) Lowest Observed Effect Conc.:

100.08

Chronic Value:

54.968

The data are normally distributed.

The data are homogenous in variance.

The number of replicates in each concentration is not equal.

The appropriate statistical test is Bonferroni's t Test. 


\section{WATER CHEYISTRY ANALYSIS}

Client: Savannah River site

Sample Identification: H - F Area

Start Date: December 12, 1990

Dilution water

ID: $\quad$ Upper Three Runs Creek water

Preparation: $\quad$ Filtered with a 37 micron Nytex mesh filter

Temperature Measurements $\left({ }^{\circ} \mathrm{C}\right)$

$\begin{array}{rrrrrrrr}\text { Day 0 } & \text { Day 1 } & \text { Day 2 } & \text { Day 3 } & \text { Day 4 } & \text { Day } 5 & \text { Day 6 } & \text { Day } 7 \\ 24.6 & 26.0 & 26.0 & 25.4 & 25.7 & 25.1 & 25.5 & 25.6\end{array}$

Conductivity Measurements (umhos)

Sample 1

Sample 2

Sample 3
49

135

1.7 
Client: Savannah River site Sample Identification: H - F Area

Start Date: December 12, 1990

Test Solutions

\section{CONTROL}

Initial

Day 1 (old)

Day 1 (new)

Day 2 (old)

Day 2 (new)

Day 3 (old)

Day 3 (new)

Day 4 (old)

Day 4 (new)

Day 5 (old)

Day 5 (new)

Day 6 (old)

Day 6 (new)

Final
$\mathrm{pH}$

D. 0 . ( $\mathrm{mg} / \mathrm{L}$

D.O.

Alkal. Hardness Conduct.

$(\mathrm{mg} / \mathrm{L}) \quad(\mathrm{mg} / \mathrm{L})$

(umhos)

6.20
6.91
6.73
6.76
6.24
6.52
6.34
6.60
6.33
6.66
6.53
6.73
6.52
6.81

$8 \cdot 3$

7.7

8.0

8.2

8.4

8.0

8.2

7.6

7.7

7.2

7.5

7.9

8.3

7.6

2

11

2

11

18

$103 \%$

$108 \%$

$100 \%$

$103 \%$

$96 \%$

$97 \%$

$90 \%$

$94 \%$

$100 \%$

$104 \%$

$96 \%$

\section{2}

3

2

3

3

3

9

\section{0\% Effluent}

Initial

Day 1 (old)

Day 1 (new)

Day 2 (old)

Day 2 (new)

Day 3 (old)

Day 3 (new)

Day 4 (old)

Day 4 (new)

Day 5 (old)

Day 5 (new)

Day 6 (o.ld)

Day 6 (new)

Final
D.O. D.O

$\mathrm{pH}$

Conductance

$(\mathrm{mg} / \mathrm{L}) \quad($ o $)$

$\begin{array}{rrrr}8.2 & 101 \% & 6.25 & 12 \\ 7.7 & 99 \% & 6.92 & \\ 8.0 & 103 \% & 6.76 & 15 \\ 7.9 & 101 \% & 6.79 & 14 \\ 8.1 & 104 \% & 6.35 & 13 \\ 7.9 & 998 & 6.62 & \\ 8.2 & 103 \% & 6.34 & 9 \\ 7.4 & 94 \% & 6.65 & 4 \\ 7.7 & 97 \% & 6.35 & \\ 6.9 & 86 \% & 6.68 & 20 \\ 7.4 & 93 \% & 6.50 & \\ 7.7 & 97 \% & 6.75 & \\ 8.1 & 103 \% & 6.37 & \\ 7.4 & 94 \% & 6.89 & \end{array}$


Client: Savannah River Site Sample Identification: H - F Area Start Date: December 12, 1990

\section{3. $0 \%$ Effluent}

Initial

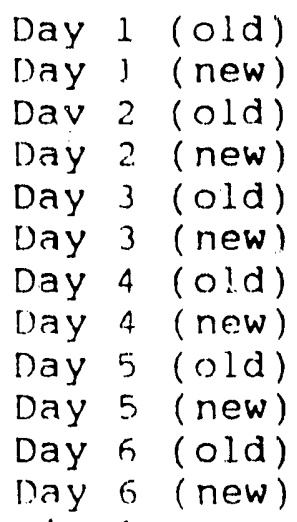

Final. $\begin{array}{ll}\text { D.O. } & \text { D.O. } \\ \left(\mathrm{mg} / \mathrm{I}_{1}\right) & \left(\frac{\%}{0}\right)\end{array} \quad$ pH Conductance

\begin{tabular}{rrrr}
8.1 & $100 \%$ & 6.29 & 14 \\
7.7 & $99 \%$ & 6.94 & \\
8.1 & $104 \%$ & 6.74 & 16 \\
7.8 & $100 \%$ & 6.81 & \\
7.9 & $101 \%$ & 6.45 & 11 \\
7.8 & $98 \%$ & 6.69 & \\
8.2 & $103 \%$ & 6.32 & 15 \\
7.0 & $89 \%$ & 6.72 & \\
7.6 & $96 \%$ & 6.38 & 12 \\
7.0 & $88 \%$ & 6.76 & \\
7.4 & $93 \%$ & 6.49 & 7 \\
7.7 & $97 \%$ & 6.81 & \\
8.1 & $103 \%$ & 6.61 & 24 \\
7.4 & $94 \%$ & 6.89 & \\
\hline
\end{tabular}

D.O. D.O. DH Conductance
$10.0 \%$ Effluent

Initjal

Day 1 (old)

Day 1 (new)

Day 2 (old)

lay 2 (new)

Day 3 (old)

Day 3 (new)

Day 4 (old)

Day 4 (new)

Day 5 (old)

Day 5 (new)

Day 6 (old)

Day 6 (new)

Final. $(\mathrm{mg} / \mathrm{L}) \quad(\%)$

8. 1

7.7

8.2

7.8

8.2

7.8

8. 2

7.2

7.6

7.1

7.4

7.7

8.1

7.3
$100 \xi$

$99 \%$

$105 \%$

$100 \%$

$105 z$

$98 \%$

$103 \%$

$91 \%$

96응

$89 \%$

93\%

$97 \%$

$103 \%$

$92 \%$
6.32

6.98

6.72

5.92

6.50

6. 78

6.36

6.79

6.38

6.79

6.47

6.83

6.63

6.94
16

19

12

24

18

7

31 


\section{QUALITY CONTROL}

Client: Savannah River site Sample Idertification: H - F Area Start Date: December 12, 1990

Culture Health:

Sensitivity: Culture organisms were exposed to $\mathrm{NaCl}$ as a reference toxicant.

$$
\begin{array}{lll}
\text { Current LC50: } & 0.593 \mathrm{~g} / \mathrm{L} \\
\text { Central Tendency: } & 0.593 \mathrm{~g} / \mathrm{L} & \\
& & \\
\text { Standard Dev: } & 0.115 & \\
\text { Upper Limit: } & 0.823 \mathrm{~g} / \mathrm{L} \quad(+2 \text { std. dev. }) \\
\text { Lower Limit: } & 0.363 \mathrm{~g} / \mathrm{L} \quad(-2 \text { std. dev.) }
\end{array}
$$

The health of the ceriodaphnia dubia used in this test were well within the normal range. 
Client: Savannah River Site $\begin{array}{lll}\text { Sample Identification: } & \text { H }- \text { F Area } \\ \text { Start Date: } & \text { December } 12,1990\end{array}$

30.0\% Effluent

Initial

Day 1 (old)

Day 1 (new)

Day 2 (old)

Day 2 (new)

Day 3 (old)

Day 3 (new)

Day 4 (old)

Day 4 (new)

Day 5 (old)

Day 5 (new)

Day 6 (old)

Day 6 (new)

Final

$100.0 \%$ Effluent

Initial

Day 1 (old)

Day 1 (new)

Day 2 (old)

Day 2 (new)

Day 3 (old)

Day 3 (new)

Day 4 (old)

Day 4 (new)

Day 5 (old)

Day 5 (new)

Day 6 (old)

Day 6 (new)

Final

\begin{tabular}{|c|c|c|c|c|c|}
\hline $\begin{array}{c}D . O \\
(\mathrm{mg} / \mathrm{L})\end{array}$ & $\left(\begin{array}{c}D . O \\
q\end{array}\right)$ & $\mathrm{pH}$ & $\begin{array}{r}\text { Alk } \\
(\mathrm{mg} / \mathrm{L})\end{array}$ & $\begin{array}{l}\text { Hard. } \\
(\mathrm{mg} / \mathrm{L})\end{array}$ & $\begin{array}{l}\text { Cond. } \\
\text { (umhos) }\end{array}$ \\
\hline 8.1 & $100 \%$ & 6.42 & - & & 22 \\
\hline 7.7 & 998 & 7.16 & - & - & \\
\hline 8.1 & $104 \%$ & 6.80 & - & 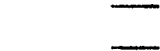 & 27 \\
\hline 7.6 & $97 \%$ & 7.16 & - & {[} & \\
\hline 8.0 & $103 \%$ & 6.67 & - & - & 17 \\
\hline 7.7 & $96 \%$ & 6.85 & - & - & \\
\hline 8.3 & $104 \%$ & 6.50 & T & T & 42 \\
\hline 7.2 & $91 \%$ & 6.89 & - & - & \\
\hline 7.7 & $97 \%$ & 6.59 & 5 & $\overline{8}$ & 10 \\
\hline 7.0 & $88 \%$ & 6.79 & & & \\
\hline 7.5 & 948 & 7.00 & 5 & 9 & 38 \\
\hline 7.8 & 998 & 6.93 & & & \\
\hline 8.3 & $105 \%$ & 6.71 & 6 & 10 & 57 \\
\hline 7.2 & $91 \%$ & 6.99 & & & \\
\hline
\end{tabular}

$\mathrm{pH} \quad(\mathrm{mg} / \mathrm{L})$

D.O. Alkal. Hardness Conduct.

$(\mathrm{mg} / \mathrm{L}) \quad(\mathrm{mg} / \mathrm{L})$ (umhos)

\begin{tabular}{|c|c|c|c|c|}
\hline $\begin{array}{l}6.97 \\
7.35\end{array}$ & $\begin{array}{l}8.2 \\
7.7\end{array}$ & $\begin{array}{r}101 \% \\
99 \%\end{array}$ & 9 & 3 \\
\hline $\begin{array}{l}7.29 \\
8.25\end{array}$ & $\begin{array}{l}8.2 \\
7.9\end{array}$ & $\begin{array}{l}105 \% \\
1018\end{array}$ & 10 & 2 \\
\hline $\begin{array}{l}7.22 \\
7.14\end{array}$ & $\begin{array}{l}8.2 \\
7.6\end{array}$ & $\begin{array}{r}105 \% \\
98 \%\end{array}$ & 9 & 4 \\
\hline 7.01 & 8.4 & 1058 & 10 & 3 \\
\hline 7.21 & 7.2 & $91 \%$ & & \\
\hline - & - & - & & \\
\hline - & - & - & & \\
\hline - & - & - & & \\
\hline - & - & - & & \\
\hline - & - & - & & \\
\hline - & - & - & & \\
\hline
\end{tabular}

49

54

44

135 

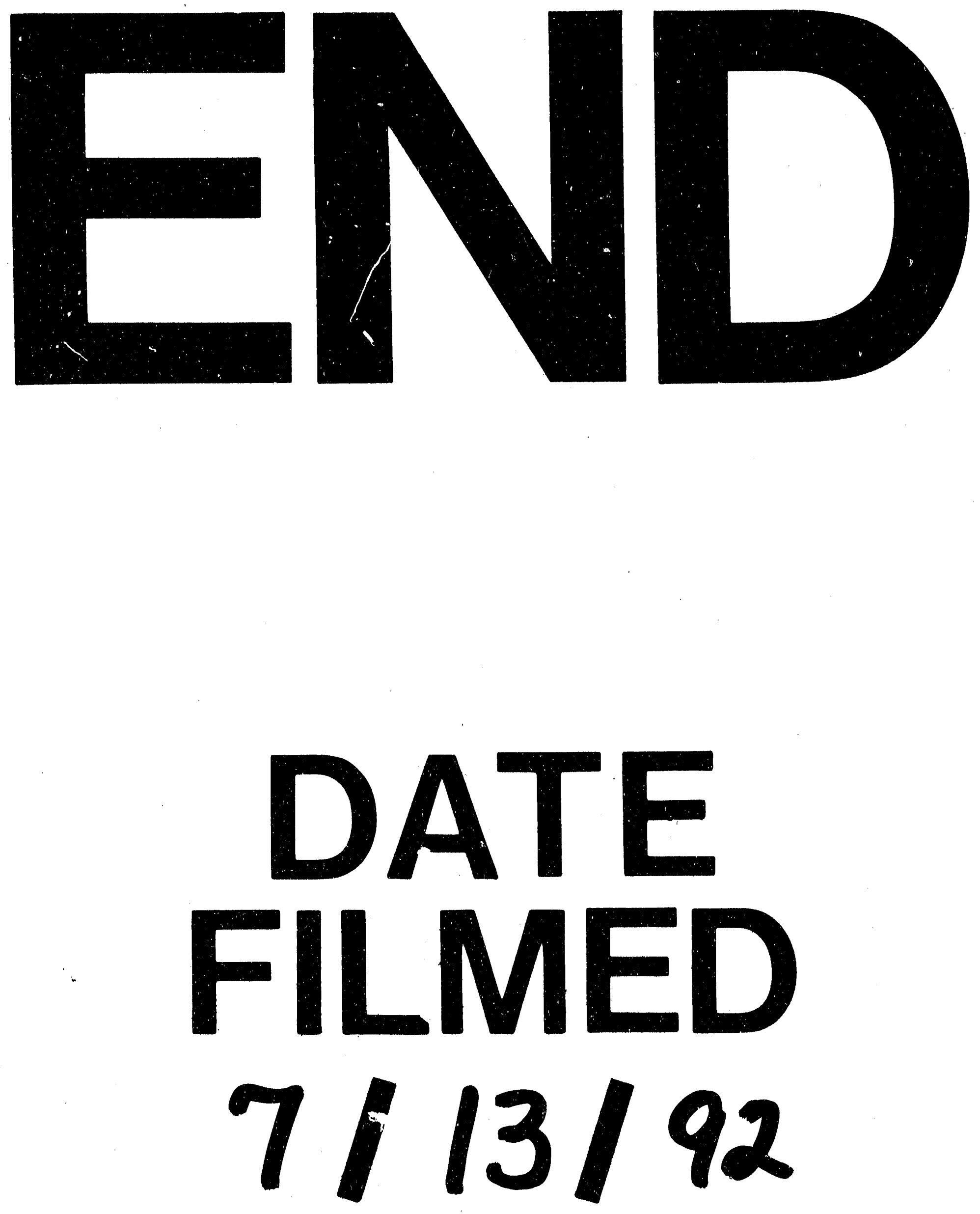
